\title{
Current Instabilities in Large-Area Silicon Diodes: An Accurate TCAD Approach
}

\author{
Luigi Balestra $^{\mathrm{a}}$, Susanna Reggiani ${ }^{\mathrm{a}}$, Antonio Gnudia ${ }^{\mathrm{a}}$, Elena Gnani ${ }^{\mathrm{a}, \mathrm{b}}$, Jan Vobecký ${ }^{\mathrm{b}, \mathrm{c}}$, \\ Umamaheswara Vemulapati ${ }^{\mathrm{b}}$ \\ ${ }^{\text {a} A R C E S ~ R e s e a r c h ~ C e n t e r ~ a n d ~ D E I, ~ U n i v e r s i t y ~ o f ~ B o l o g n a, ~ B o l o g n a, ~ I t a l y ~}$ \\ ${ }^{b}$ Hitachi-ABB Power Grids, CH-5600 Lenzburg, Switzerland \\ 'Microelectronics Department, Czech Technical University in Prague, CZ-166 27 Prague 6, Czech Republic \\ luigi.balestra5@unibo.it
}

\begin{abstract}
Fast-recovery diodes can exhibit negative differential resistance during reverse recovery which is associated with the formation of current filaments triggered by the presence of inhomogeneities along the device. Physics concerning the dynamics of current filaments has been widely analyzed in literature through numerical simulations. However, a method to clearly identify the elements that dominate on the current pattern formation is still not available since filaments can appear also in uniform structures. In this work, a novel TCAD approach is provided to suppress the numerical errors due to the discretization of the transport equations which allows us to analyze the effects of the junction depth and the presence of different doping inhomogeneities in the anode doping profile of large-area diodes.
\end{abstract}

Keywords: Fast-recovery diode, Reverse recovery, Current filaments, Numerical simulations

\section{Introduction}

Fast-Recovery Diodes (FRD) play a fundamental role the state-of-the-art power converters. Any active switch adopted for power conversion, such as MOSFET, IGBT and IGCT requires a complementary free-wheeling FRD [1] [2]. The circuit with IGCTs require also clamping FRD. All these FRDs have to cope with high dynamic stress during the turn-off of inductive load. That is, they must be able to safely turn off even with very high $d I / d t$ rate and high DC link voltage $V_{D C}$. During the switching process the so-called dynamic avalanche condition occurs: the charge carriers extracted from the plasma cross the space charge region of a blocking junction and increase the effective positive/negative charge hereby enhancing the electric field peaks [3]. For this reason, the effective breakdown voltage (BV) during the reverse recovery transient can be significantly lower than the static BV, leading to current instabilities [4] [5] [6]. During the reverse recovery, diodes can exhibit a negative differential resistance which is correlated with the inhomogeneous current density distribution along the device [7] [8]. The consequent current filamentation may lead to the destruction of the device [9] [10] [11]. The physical mechanism concerning the pattern formation have been successfully analyzed through numerical simulations in several works. Even if the non-linear dynamics of current filamentation was nicely investigated in the previous works, the fundamental origin of the instability was not addressed. As it is known, the current filaments can be easily caused by the numerical errors in the discretization of the transport equations, providing the formation of current filaments in perfectly uniform structures, as e.g. in [7] [8]. Under such conditions, it becomes very difficult to study how the different nonhomogeneities which may arise in a real device contribute to the formation of current filaments [12]. In this work, a method to suppress the numerical error and to clearly distinguish fluctuation due to the discretization of transport equation from those coming from a nonhomogeneity in the device is provided and applied to 4.5kV FRD [13].

\section{TCAD setup}

Numerical simulations are a useful tool in the analysis of current fluctuations in power diodes under reverse recovery conditions. It is well known that the presence of any kind of non-homogeneity can be the starting point for the formation of current filaments. It is then fundamental to find a precise TCAD setup minimizing the nonhomogeneous distribution of the numerical errors so as to allow for the study of specific physical inhomogeneities in the device.

\section{Device used for simulations}

The simulated device is the FRD with a wafer thickness of $660 \mu \mathrm{m}$. The effective device area is in the order 


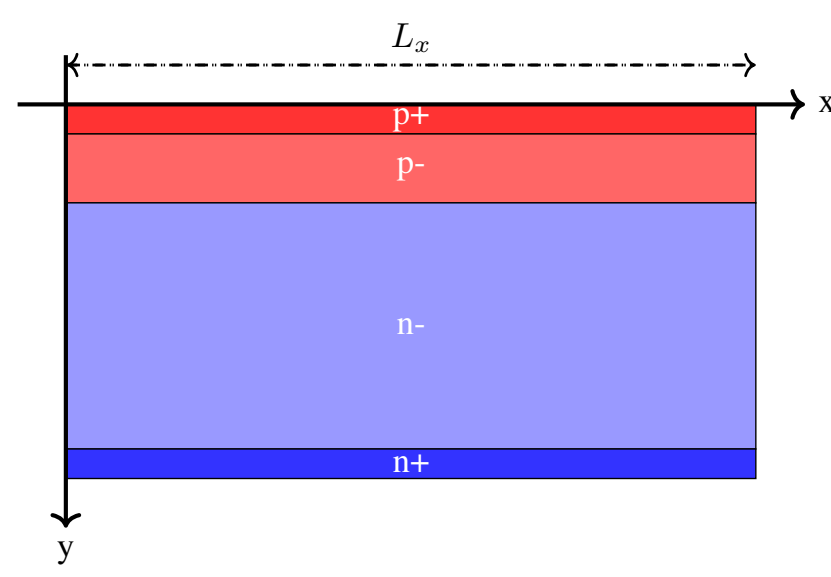

Fig. 1. Schematic representation of the power diode

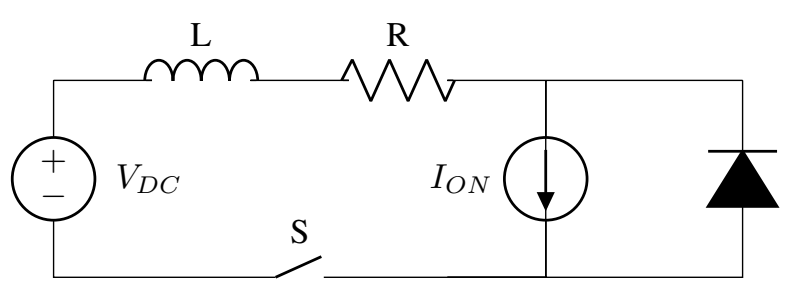

Fig. 2. Test circuit used for reverse recovery simulations. $V_{D C}=$ $2800 \mathrm{~V}, I_{O N}=2500 \mathrm{~A}, L=325 n \mathrm{H}, R=R_{0} e^{-t / \tau}$ with $R_{0}=$ $23 \Omega$ and $\tau=0.75 \mu \mathrm{s}$

of $10^{8} \mu \mathrm{m}^{2}$ regardless of the lateral width $L_{x}$ of the structure. Device simulations have been performed by the drift-diffusion transport equations in two dimensional (2D) structure under transient condition [14]. The SRH recombination has been accounted for with lifetimes equal to $\tau_{e}=\tau_{h}=1 \mathrm{~ms}$. Avalanche generation (UniBo impact ionization model), Auger recombination, mobility doping dependence and carrier-carrier scattering have been accounted for with default parameters. Default values of the surface recombination velocities have been used at the Aluminum/Silicon interfaces. Iso-thermal conditions have been assumed with a fixed ambient temperature of $\mathrm{T}=413 \mathrm{~K}$. A schematic view of the device is shown in Fig. 1. The circuit used to simulate the reverse recovery transient is reported in Fig. 2 along with the corresponding component values corresponding to real power converter.

\section{Meshing Criteria}

In order to limit the current instabilities due to nonhomogeneous meshing of the device, a specific grid has been created to simulate the FRD under reverse recovery transient. In this section the criteria adopted to generate the grid are given. Starting from the uniform diode we checked that the proposed meshing criteria would give rise to grids with negligible current fluctuations as expected when an ideal device is used. The lateral width of the device is fixed at $L_{x}=1000 \mu \mathrm{m}$. A uniform rectangular grid geometry was chosen because, differently from a triangular mesh, it reproduces the

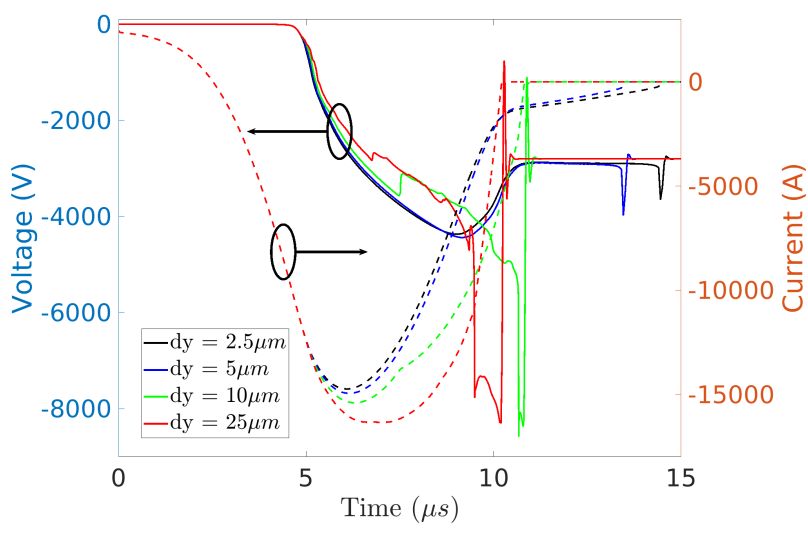

Fig. 3. $V(t), I(t)$ for the same device but with different mesh spacing in the drift region

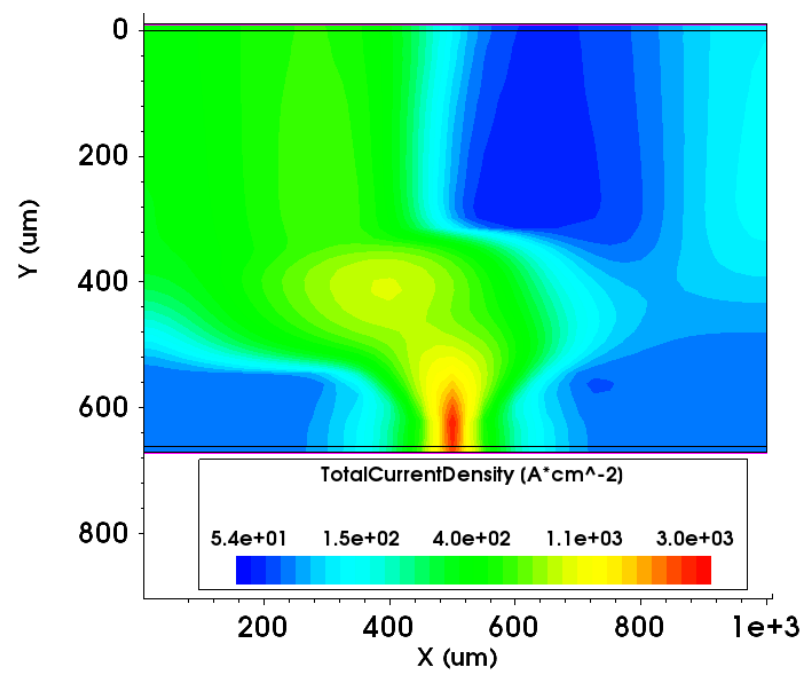

Fig. 4. 2D contour plot of the simulations with mesh-spacing dy $=$ $25 \mu \mathrm{m}$ at $\mathrm{t}=7.41 \mu \mathrm{s}$ showing the presence of cathode-side current filaments.

homogeneity of the current distribution as shown in [12]. The grid used to simulate the diode was defined as follows. A uniform spacing along the $\mathrm{x}$-axis $(d x)$ is fixed with $d x=25 \mu \mathrm{m}$. Differently, in the vertical direction the $\mathrm{p}-\mathrm{n}$ junction requires to adopt fine grid spacing close to the $\mathrm{p}-\mathrm{n}$ junction and near the electrode. To this purpose the spacing along the y-axis $(d y)$ is $1 \mu \mathrm{m}$ for all simulations. Different $d y$ in the drift region have then been tested. In Fig. 3, the simulated voltage and current curves for the FRD in reverse recovery conditions are reported for different vertical grid space. The $\mathrm{V}(\mathrm{t})$ curves of structures with larger dy $(10 \mu \mathrm{m}$ and $25 \mu \mathrm{m})$ show significant voltage overshoots, which correspond to the presence a of moving anode filaments and static cathode-side filament in the middle of the device as shown in Fig. 4. In the case of shorter $d y$, smooth and regular curves were observed for both $d y=5 \mu \mathrm{m}$ and $d y=2.5 \mu \mathrm{m}$. However, by monitoring the maximum current contribution to current density in the x-direction during reverse recovery, which should be ideally zero due to the uniformity of the p-n junction, a significantly higher contribution was found for $d y>2.5 \mu \mathrm{m}$ as shown 

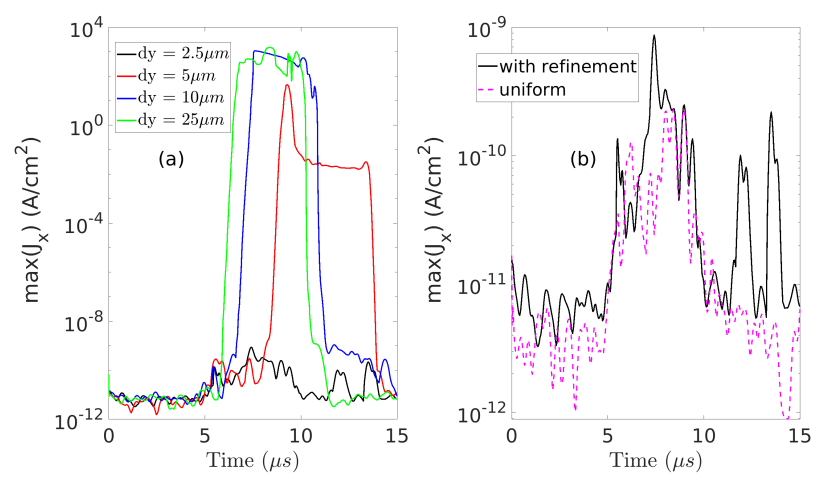

Fig. 5. Maximum value of the current density x-component as function of time. (a) $\max \left(J_{x}\right)$ corresponding to simulations of Fig. 3. (b) both simulations have $d y=2.5 \mu \mathrm{m}$ in the drift region. Black solid line: with refinement $(d y=1 \mu m)$ at the $\mathrm{p}-\mathrm{n}$ junction and at the contact. Magenta dashed line: with $d y=2.5 \mu \mathrm{m}$ everywhere.
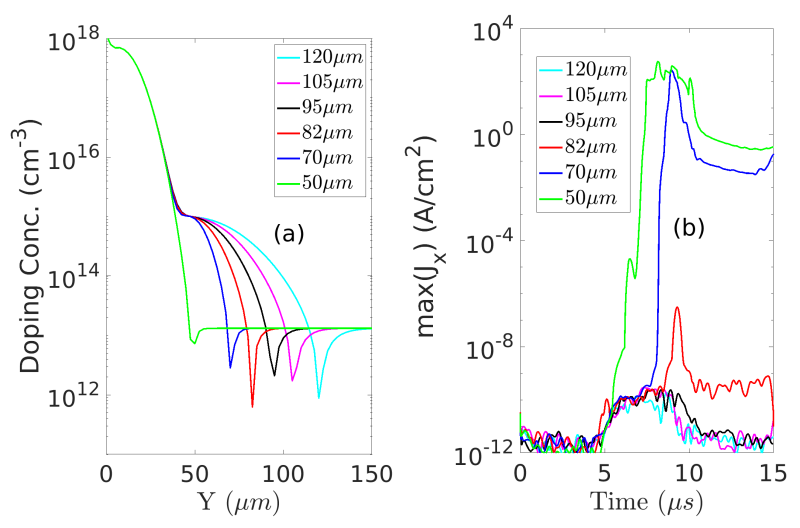

Fig. 6. (a) Anode doping profile with different junction depth $\left(y_{J}\right)$. (b) Maximum value of $J_{x}$ as function of time.

in Fig. 5(a). This led to the choice of the best $d y$ in the drift region, which gives a numerical error on the current fluctuations comparable to the case of a uniform grid as shown in Fig. 5(b). In conclusion, the most suitable setup for the reverse recovery simulations of power diodes consists of a uniform rectangular grid with spacing along the y-axis small enough to suppress as much as possible what needs to be checked by monitoring the $\mathrm{x}$-component of the current density.

\section{Factors enhancing the generation of current} filaments

With the setup described above, we will further investigate three different factors that can cause pattern formations.

\section{Anode doping profile}

Simulations of the FRD with different anode doping profiles have been carried out as shown in Fig. 6 (a). The depth of the p-n junction, $y_{J}$, has been chosen so as to have the same static breakdown conditions due to the punch through, but, viceversa, to strongly affect the electric field peak at the $p-n$ junction leading to a larger impact ionization in dynamic conditions. This is

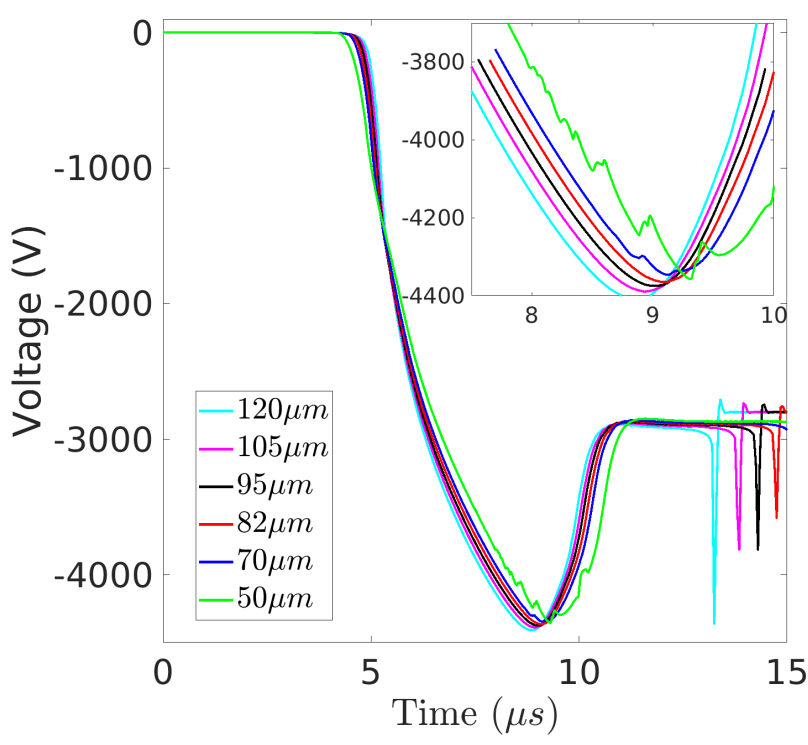

Fig. 7. $\mathrm{V}(\mathrm{t})$ reverse recovery curves of diode with the doping profile shown in Fig. 6(a).Inset: zoomed curves in the time range from 7.5 to 10us.

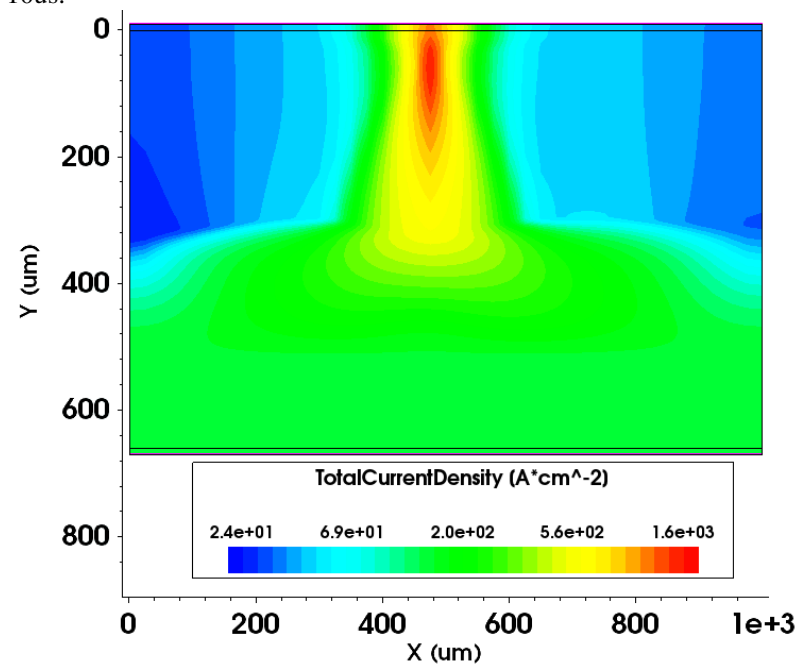

Fig. 8. 2D contour plot of the diode with the $50 \mu m$ deep p-base from Fig. 6(a) at $\mathrm{t}=8.65 \mu \mathrm{s}$ showing the presence of anode-side current filaments.
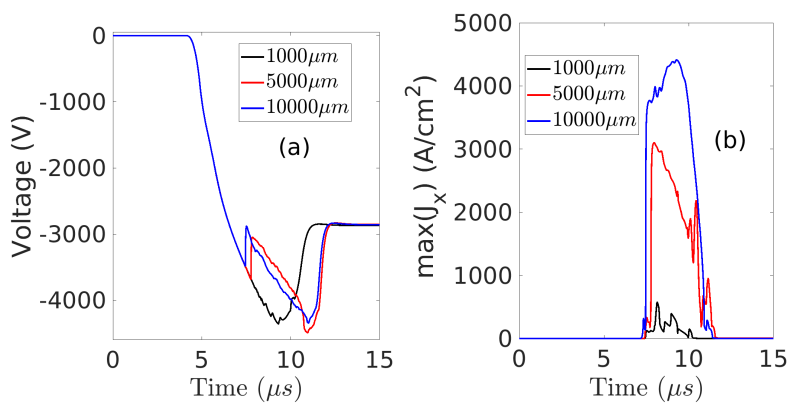

Fig. 9. (a) $\mathrm{V}(\mathrm{t})$ curves of the diode with $50 \mu \mathrm{m}$ deep anode doping profile and different lateral width $L_{x}$ (b) Maximum value of $J_{x}$ corresponding to simulations shown in (a).

clearly shown in Fig. 6 (b) where the $J_{x}$ fluctuations are compared for the different $y_{J}$. No significant fluctuations are observed for $y_{j} \geq 95 \mu \mathrm{m}$. On the contrary, there is no way to suppress current fluctuations for lower $y_{j}$, 
even if the device is ideally uniform. This gives rise to current filaments occurring in the time range from 7.5 and $10 \mu \mathrm{s}$, as shown in Figs. 7 and 8. By using this kind of simulations, the smallest value of $y_{j}$ providing high ruggedness to dynamic turn-off can be extracted.

\section{Lateral width of the simulated device}

It is known form literature [15] that instabilities can easily develop into current filaments if the device has large lateral dimension $L_{x}$. This can be shown by performing simulations of the same diode but with different $L_{x}$. In Fig. 9(a), simulations of three diodes with $y_{J}=50 \mu \mathrm{m}$ and lateral lengths $L_{x}=1000,5000$ and $10000 \mu \mathrm{m}$ are compared. The shortest one corresponds to the reference diode used in the analysis of the anode doping profile which gives rise to the anode current filament shown in Fig. 8. The comparison of the $V(t)$ curves shows, that the significant voltage peaks in the $\mathrm{V}(\mathrm{t})$ of the diodes with larger Lx (Fig. 9(a)) correspond to the sudden increases of JX (Fig. 9(b)) manifested by the formation of a cathode-side filament in addition to the anode-side one (not shown). This result is in good agreement with expected theoretical results and experimental evidence as well.

\section{Doping Fluctuations}

Small doping inhomogeneities in the anode profile, which are common in all power diodes [16], are suspicious to play a role in the onset of current instabilities. In order to investigate the effect of such non-uniformities on the generation of current filaments, a single doping inhomogeneity has been added in the middle of the diode structure with $y_{J}=95 \mu \mathrm{m}$ and $L_{x}=1000 \mu \mathrm{m}$, which is one of the stable structures in Fig. 6. The lateral extension of the inhomogeneity was fixed to $100 \mu \mathrm{m}$, while different possible profile shapes of a potential nonuniformity have been tested as shown in Fig. 10 (a). The corresponding magnitudes of the maximum $J_{x}$ reported in Fig. 10 (b) clearly show that the worst case is given by the doping profiles with locally lowered concentration (B1 and B2) contrary to the increased one (A1 and A2). Even if no filaments were generated by such fluctuations, a larger instability with respect to the homogeneous reference case is found, which might be the cause of the onset of instability, if other factors promoting the instabilities discussed above take place.

\section{Conclusion}

In this work, a method to suppress the numerical error in reverse recovery simulations of power diodes has been provided to precisely analyze the formation of current filaments through TCAD simulations. Analysis of current fluctuations along the lateral direction shows that a rectangular mesh with uniform spacing along the $\mathrm{x}$ and $\mathrm{y}$ axis is the most suitable one to prevent the formation of current filaments in a uniform structure. Such method has been applied to study the factors
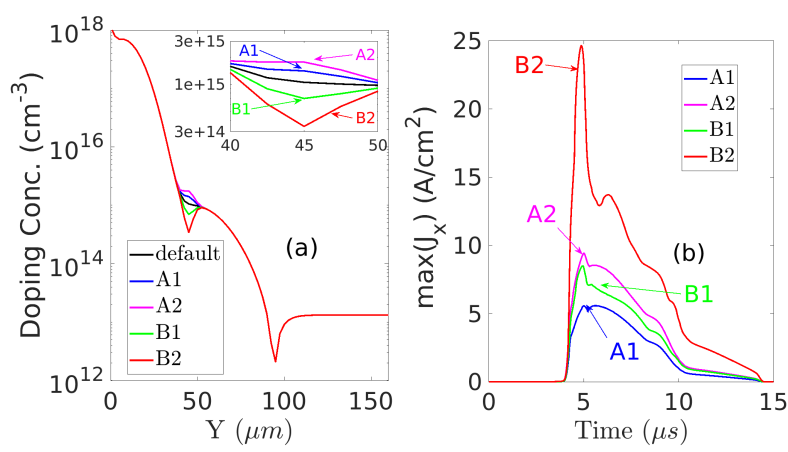

Fig. 10. (a) Vertical doping profile with small inhomogeneities (A1, A2, B1, B2) magnified in the inset (b). Maximum value of $J_{x}$ corresponding to the simulations shown in (a).

that enhance the probability of filaments generation. A criterion to find the smallest value of the junction depth that provides a high dynamic ruggedness has been described. Theoretical predictions about the role of lateral dimension are confirmed by simulations performed with different values of $L_{x}$. In addition, the effects of different doping inhomogeneities has been considered showing how region with locally lower doping concentration are more susceptible to current instabilities. The proposed approach is a solid starting point for a rigorous analysis of the impact of different inhomogeneities, which may appear especially in the real large-area high-voltage fast recovery diode, hereby providing a useful tool for further enhancement of their ruggedness and reliability.

\section{References}

[1] Baliga, B. Jayant. Fundamentals of power semiconductor devices. Springer Science \& Business Media, 2010.

[2] Mohan, Ned. Power electronics: a first course. Wiley, 2011.

[3] Lutz, J., 1999. Stable dynamic avalanche in Si power diodes. Applied physics letters, 74(21), pp.3170-3172. Lutz, J. and Domeij, M., 2003. Dynamic avalanche and reliability of high voltage diodes. Microelectronics Reliability, 43(4), pp.529-536.

[4] Domeij, M., Breitholtz, B., Östling, M. and Lutz, J., 1999. Stable dynamic avalanche in $\mathrm{Si}$ power diodes. Applied physics letters, 74(21), pp.3170-3172.

[5] Domeij M, Breitholtz B, Hillkirk LM, Linnros J, Ostling M. Dynamic avalanche in 3.3-kV Si power diodes. IEEE transactions on Electron Devices. 1999 Apr;46(4):781-6.

[6] Domeij, M., Lutz, J. and Silber, D., 2003. On the destruction limit of Si power diodes during reverse recovery with dynamic avalanche. IEEE Transactions on Electron Devices, 50(2), pp.486493.

[7] Niedernostheide, F.J., Falck, E., Schulze, H.J. and KellnerWerdehausen, U., 2004. Current-density patterns induced by avalanche injection phenomena in high-voltage diodes during turn-off. Annalen der Physik, 13(7-8), pp.414-422.

[8] Niedernostheide, F.J. and Schulze, H.J., 2004. Studies on dynamic avalanche and current filaments in high-voltage diodes. Physica D: Nonlinear Phenomena, 199(1-2), pp.129-137.

[9] Baburske, R., Heinze, B., Lutz, J. and Niedernostheide, F.J., 2008. Charge-Carrier Plasma Dynamics During the Reverse-Recovery Period in $\mathrm{p}^{+}-\mathrm{n}^{-}-\mathrm{n}^{+}$Diodes. IEEE transactions on electron devices, 55(8), pp.2164-2172.

[10] Baburske, R., Heinze, B., Niedernostheide, F.J., Lutz, J. and Silber, D., 2009, June. On the formation of stationary destructive cathode-side filaments in $\mathrm{p}^{+}-\mathrm{n}^{-}{ }^{-} \mathrm{n}^{+}$diodes. In 2009 21st International Symposium on Power Semiconductor Devices \& IC's (pp. 41-44). IEEE.

[11] Baburske, R., Niedernostheide, F.J., Lutz, J., Schulze, H.J., Falck, E. and Bauer, J.G., 2013. Cathode-side current filaments in high- 
voltage power diodes beyond the SOA limit. IEEE transactions on electron devices, 60(7), pp.2308-2317.

[12] Shiba, Y., Omura, I. and Tsukuda, M., 2016, June. IGBT avalanche current filamentaion ratio: Precise simulations on mesh and structure effect. In 2016 28th International Symposium on Power Semiconductor Devices and ICs (ISPSD) (pp. 339-342). IEEE.

[13] S. Reggiani et al., "TCAD Investigation of Differently-Doped DLC Passivation for Large-Area High-Power Diodes," in IEEE Journal of Emerging and Selected Topics in Power Electronics, doi: 10.1109/JESTPE.2019.2921871.

[14] Synopsys Inc., Sentaurus Device User Guide M-2016.12, 2016.

[15] Gorbatyuk, A.V. and Niedernostheide, F.J., 1999. Mechanisms of spatial current-density instabilities in $p^{+}-p^{-}-n-p^{+}-n^{++}$ structures. Physical Review B, 59(20), p.13157.

[16] Kwok, D.T.K., Chu, P.K., Wood, B.P. and Chan, C., 1999 Particle-in-cell and Monte Carlo simulation of the hydrogen plasma immersion ion implantation process. Journal of applied physics, 86(4), pp.1817-1821. 